\title{
Composición química y actividad antioxidante del alga marina roja Bryothamnion triquetrum (S.G.Gmelin) Howe
}

\author{
Alexis Vidal ${ }^{1}$, Adyary Fallarero' ${ }^{1}$ Elma Regina Silva de Andrade-Wartha ${ }^{2}$, Ana Mara de Oliveira e \\ Silva ${ }^{2}$, Alessandro de Lima², Rosângela Pavan Torres², Pia Vuorela ${ }^{3}$, Jorge Mancini-Filho ${ }^{2}$ \\ ${ }^{1}$ Departamento de Bioquímica, Facultad de Biología, Universidad de La Habana, ${ }^{2}$ Departamento de Alimentos e \\ Nutrição Experimental, Faculdade de Ciências Farmacêuticas, Universidade de São Paulo, ${ }^{3}$ Departamento de \\ Bioquímica y Farmacia, Facultad de Matemática y Ciencias Naturales, Åbo Akademi University
}

*Correspondência:

J. Mancini-Filho

Departamento de Alimentos e

Nutrição Experimental

Facultade de Ciências Farmacêuticas Universidade de São Paulo

Av. Prof. Lineu Prestes 580, Bloco 14 05508-900 - São Paulo-SP, Brasil

E-mail: jmancini@usp.br
En la actualidad existe un marcado interés por la búsqueda de antioxidantes de fuentes naturales, incluidas las algas marinas. El objetivo de este trabajo fue evaluar la composición química y propiedades antioxidantes del alga Bryothamnion triquetrum. Se estudió la composición centesimal y de minerales, identificación de ácidos grasos y sustancias antioxidantes. La composición centesimal es la siguiente: Proteinas (9,5\%), Lipidos (1,3\%), Carbohidratos (5,9\%), Fibras (10,2\%) y Cenizas (43\%). Los resultados de la actividad antioxidante para las diferentes metodologías empleadas fueron: atrapamiento de radicales DPPH (38\%, $4 \mathrm{mg}$ de liofilizado), $\beta$-Caroteno-Linoleico (12\%, $4 \mathrm{mg}$ de liofilizado), actividad atrapadora de radicales $\mathrm{O}_{2}^{-}\left(\mathrm{CI}_{50} 0,36 \mathrm{mg} /\right.$ $\mathrm{mL})$, de radicales $\mathrm{OH}^{*}\left(\mathrm{CI}_{50} 2,11 \mathrm{mg} / \mathrm{mL}\right)$ y unión al $\mathrm{Fe}\left(\mathrm{CI}_{50} 0,37\right.$ $\mathrm{mg} / \mathrm{mL}$ ). Las propiedades antioxidantes de esta alga parecen explicarse por la capacidad atrapadora de radicales libres, particularmente relacionada con mecanismos de dismutación de radicales $\mathrm{O}_{2}^{-}$, inactivación de radicales $\mathrm{OH}^{\circ}$ y quelación de Fe. En trabajos previos se identificaron ácidos cinámicos y fenólicos como moléculas que pudieran explicar la actividad antioxidante, sin embargo adicionalmente se debe considerar un efecto sumatorio y/o sinérgico de otros componentes antioxidantes del extracto, como los descritos en este trabajo, incluidos minerales, carotenoides y vitamina $C$.

\section{INTRODUCCIÓN}

En los últimos años se ha incrementado el interés por la búsqueda de antioxidantes naturales, generalmente constituidos por mezclas de compuestos con una elevada diversidad molecular y funcionalidad biológica (Aruoma et al., 2003). En su mayoría estas entidades son aceptadas como no tóxicas, aunque algunos de estos compuestos 
naturales pudieran presentar un definido riesgo toxicológico (Vidal et al., 2005).

Las algas marinas son consideradas como un alimento tradicional en la dieta de diferentes pueblos, sobretodo asiáticos; de acuerdo a su composición son alimentos bajos en calorías, con altas concentraciones de minerales, vitaminas y proteínas, muy ricos en fibras y relativamente altas concentraciones de ácidos grasos poli-insaturados (Jimenez-Escrig, Goñi,1999; Risso et al.,2003). En su mayoría se localizan en la zona fótica, con una notable exposición a la radiación solar, lo que favorece la generación de radicales libres, y esto a su vez pudiera condicionar la biosíntesis de metabolitos secundarios con propiedades antioxidantes. Se sugiere que la ausencia de daño oxidativo en los componentes estructurales de las algas y su estabilidad frente a determinadas condiciones adversas se deben a la presencia de efectivos antioxidantes (Burritt et al., 2002).

En diferentes investigaciones se ha demostrado una relación directa entre el consumo de algas marinas y la prevención y/o en el tratamiento de patologías relacionados con el estrés oxidativo (Funahashi et al., 2001; Jiménez-Escrig, Sánchez-Muñiz, 2000; Yuan, Walsh, 2006). Algunos autores han comprobado que extractos de algas marinas tienen actividad antioxidante explicada por disímiles mecanismos de acción, incluídos la capacidad atrapadora de radicales libres, la quelación de metales activos desde el punto de vista redox, los mecanismos de donación y aceptación de electrones, la capacidad de interrupción de la peroxidación lipidica y el incremento de la actividad de enzimas antioxidantes (Yan et al., 1998, 1999; Jiménez-Escrig et al., 2001; Lim et al., 2002). Esta actividad a su vez pudiera explicar las propiedades neuroy hepato-protectoras que presentan algunos de estos extractos (Linares et al., 2004; Raghavendran et al., 2005).

El alga marina Bryothamnion triquetrum crece rápidamente a poca profundidad, es fácil de recolectar, no es tóxica y se encuentra ampliamente distribuida (Areces,1995), aspectos todos que avalan sus potencialidades como fuente de compuestos bioactivos. Las actividades antioxidante y neuroprotectora del alga $B$. triquetrum han sido estudiadas con diferentes procedimientos experimentales in vitro y en cultivos de células neuronales GT1-7 y ha sido relacionada con los ácidos fenólicos y cinámicos identificados en este extracto, sin embargo aun no ha sido esclarecido completamente su mecanismo de acción (Vidal et al., 2001; Fallarero et al., 2002, 2003, 2006).

Considerando estos antecedentes, el objetivo de este trabajo fue evaluar la composición química y las propiedades antioxidantes del alga marina Bryothamnion triquetrum y a la vez profundizar en los mecanismos de acción de esta propiedad. Los resultados de este trabajo avalan sus potencialidades como fuente natural de antioxidantes con perspectivas de aplicaciones como fitofarmaco y/o suplemento dietético funcional.

\section{MATERIALES Y MÉTODOS}

\section{Colecta de algas}

El alga marina roja Bryothamnion triquetrum (S.G.Gmelin) Howe fue colectada en el área del Bajo de Santa Ana (La Habana, Cuba) en agosto/2005. Los especimenes fueron identificados y autenticados en el Laboratorio de Algas del Centro de Investigaciones Marinas de la Universidad de La Habana, donde se depositó una muestra.

\section{Preparación de los extractos}

Los ejemplares frescos de B.triquetrum se lavaron y se homogeneizaron en $\mathrm{H}_{2} \mathrm{O}$ destilada manteniendo una relación 1:4 (p/v). Los homogenados B.triquetrum obtenidos se centrifugaron a $800 \mathrm{~g},-4{ }^{\circ} \mathrm{C}$ durante 20 minutos. Los sobrenadantes fueron liofilizados y posteriormente conservados a $-20{ }^{\circ} \mathrm{C}$ hasta su uso. El rendimiento estimado del extracto final liofilizado en comparación con el alga fresca fue de $0,5 \%$.

\section{Composición centesimal}

Las algas recién colectadas se lavaron con $\mathrm{H}_{2} \mathrm{O}$ destilada y se dejaron secar bajo sombra a temperatura ambiente. Posteriormente porciones de algas secas fueron molidas en un molino eléctrico (IKA A11 Basic) y tamizadas a 32 mesh. Las determinaciones de humedad, cenizas, proteínas, lípidos totales y fibras se realizaron de acuerdo a los procedimentos establecidos por la AOAC (1995). En todos los casos, las determinaciones se realizaron por triplicado.

\section{Preparación de la fracción lipidica}

A porciones de algas secas y molidas se les realizó una extracción de la fracción lipídica según el procedimiento de Folch et al. (1957): porciones de 5 gramos de alga se extrajeron con metanol $(50 \mathrm{~mL})$, y posteriormente con cloroformo $(100 \mathrm{~mL})$, entonces se le adicionaron $\mathrm{KCl}$ al $0,88 \%$ ( $1 / 4$ del volumen). La fase orgánica se eliminó por rotoevaporación y se redisolvió en 
$5 \mathrm{~mL}$ de cloroformo. Se le determinó la cantidad de solutos totales por gravimetría $\left(1 \mathrm{~mL} / 105^{\circ} \mathrm{C}\right)$.

Los ácidos grasos de la fracción lipídica se metilaron de acuerdo al procedimiento de Hartman y Lago (1973), empleando ácido heptadecanoico como estándar interno; a las muestras lipidicas se le adicionó KOH 0,5 N (2 mL) en metanol, se calentó a $100{ }^{\circ} \mathrm{C}(5 \mathrm{~min})$ en tubos cerrados herméticamente, para agregar más adelante $(6 \mathrm{~mL})$ la mezcla de metilación $\left(\mathrm{NH}_{4} \mathrm{Cl}\right.$ en $\left.\mathrm{MeOH}: \mathrm{H}_{2} \mathrm{SO}_{4}\right)$, se calentó a $100{ }^{\circ} \mathrm{C}$ (3 min). Los ácidos grados metilados se extrajeron con hexano, la fase orgánica se llevó a sequedad y se redisolvió en hexano. A cada fracción se le determinó la cantidad de solutos totales por gravimetría.

\section{Identificación y cuantificación de los ácidos grasos}

Alícuotas de los ácidos grasos metilados fueron estudiados mediante cromatografía gaseosa de acuerdo al procedimiento reportado por AOAC (2002). Muestras de ácidos grasos metilados se analizaron en un CG Shimadzu QP 5000 equipado con detector de ionización de llama y una columna capilar de sílica fundida Supelcowax 10 (J\& W Scientific) (30 $\mathrm{m} \times 0,25 \mathrm{~mm} \times 0,25 \mu \mathrm{m})$. Las temperaturas del inyector y el detector fueron 250 y $270{ }^{\circ} \mathrm{C}$, respectivamente. El programa de temperatura fue: $170{ }^{\circ} \mathrm{C}$ a $225^{\circ} \mathrm{C}$, con un gradiente de $1{ }^{\circ} \mathrm{C} / \mathrm{min}$. El gas portador fue helio con un flujo de $1 \mathrm{~mL} / \mathrm{min}$. El AUC de cada pico fue analizado mediante un integrador GC Workstation ClassGC-10 (Shimadzu).

\section{Análisis cuantitativo de la composición de minerales}

El análisis de la composición de minerales se realizo de acuerdo al método de espectroscopía atómica descrito por Saari y Paaso (1980). Las muestras del extracto liofilizado, se prepararon con una digestión húmeda con $\mathrm{HNO}_{3}$ concentrado para posteriormente realizar la determinación con un espectrómetro de masas Perkin Elmer Sciex Elan 6000 (Perkin Elmer, USA). Adicionalmente el contenido de Se fue determinado mediante un método de absorción electrotérmica atómica descrito por Kumpulainen et al. (1983). Las muestras (1 g) fueron digeridas en una mezcla de $\mathrm{HNO}_{3}, \mathrm{HClO}_{4} \mathrm{y}$ $\mathrm{H}_{2} \mathrm{SO}_{4}(1: 1: 1)(20 \mathrm{~mL})$ y luego tratadas para reducir todo el selenio a selenio (IV), el que luego fue quelado com pirrolidinditiocarbamato de amonio y extraído en una fase metil-isobutil-cetona $(20 \mathrm{~mL})$, para la determinación en un espectrofotómetro de absorción atómica modelo 5100 Zeeman (Perkin Elmer, USA). Como estándar externo y para el control de calidad se emplearon una solución estándar Merck de multi- elementos en concentraciones de 10 mg.L - $^{-1}$ (10580 ICP MultiElement Standard VI) y muestras certificados de tejido de ostras, NBS 1566a (NIST, USA) y harina de trigo NBS 1567a (NIST, USA).

\section{Determinación del contenido de ácido ascórbico y carotenoides}

Se realizó según la metodología espectrofotométrica propuesta por Natelson (1964), que utiliza el reactivo de 2,4-dinitrofenilhidracina-tiourea. Se empleó como estándar una solución de ácido ascórbico a la concentración de $0,01 \mathrm{mg} / \mathrm{mL}$.

La determinación del contenido total de carotenos se realizo de acuerdo a la metodología propuesta por la AOAC (1990): extracción con acetona: hexano $(1: 1,5)$ y purificación en una columna de $\mathrm{MgO}$. La calibración del método fue realizada con $\beta$-caroteno (Fluka, E.U.) y los resultados fueron expresados como mg de equivalentes de $\beta$-caroteno/g de extracto liofilizado.

\section{Determinación de la actividad antioxidante}

Ensayo de actividad atrapadora de radicales 1,1-difenil2-picrilhidracil (DPPH')

Este ensayo se realizó según el protocolo descrito por Goupy et al. (1999). 0,5 mL del extracto liofilizado (en las concentraciones de 500-8000 $\mu \mathrm{g}$ ) de B.triquetrum disuelto en $\mathrm{H}_{2} \mathrm{O}$ destilada se agregó a una solución metanólica de DPPH$(1,5 \mathrm{~mL})$, empleando como control positivo trolox $50 \mu \mathrm{M}$. La absorbancia de la solución del radical estable en presencia de la muestra $\left(\mathrm{A}_{\mathrm{m}}\right)$ fue medida en un espectrofotómetro (Spectronic instruments, USA) a $517 \mathrm{~nm}$. Los porcentajes de inhibición (PI) de la absorción del $\mathrm{DPPH}^{*}$ producidos por B.triquetrum fueron calculados en relación con la absorbancia de la muestra blanco $\left(\mathrm{A}_{b}\right.$, con $\mathrm{H}_{2} \mathrm{O}$ destilada) usando la siguiente ecuación: $\mathrm{PI}=\left[\left(\mathrm{A}_{\mathrm{b}}-\mathrm{A}_{\mathrm{m}}\right) / \mathrm{A}_{\mathrm{b}}\right] \times 100 . \mathrm{La} \mathrm{CI}_{50}$ fue calculada según la relación: $\mathrm{CI}_{50}=\mathrm{C}_{1}-\Delta \mathrm{C}$, donde: $\Delta \mathrm{C}=\left[\left(\mathrm{C}_{1}-\mathrm{C}_{2}\right) \mathrm{x}\right.$ $\left.\left(\mathrm{PI}_{1}-50\right)\right] /\left(\mathrm{PI}_{1}-\mathrm{PI}_{2}\right)$, en el que: $\mathrm{PI}_{1}$ y $\mathrm{PI}_{2}$ corresponden a los valores de porcentajes de inhibición inmediatamente superiores e inferiores al $50 \%$ de inhibición y $\mathrm{C}_{1}$ y $\mathrm{C}_{2}$ corresponden a las concentraciones en las que se producen $\mathrm{PI}_{1}$ y $\mathrm{PI}_{2}$, respectivamente.

Actividad antioxidante con el ensayo del $\beta$-Caroteno-ácido linoléico

La actividad antioxidante fue evaluada por el procedimiento de Miller (1971): una mezcla $\beta$-caroteno $(20 \mu \mathrm{L})$, ácido linoléico $(40 \mu \mathrm{L})$ y Tween $40(530 \mathrm{mg})$ se 
suspendieron en agua oxigenada $(100 \mathrm{~mL})$. Porciones de esta emulsión $(5 \mathrm{~mL})$ fueron colocados en diferentes tubos conteniendo muestras $(200 \mu \mathrm{L})$ o control positivo butilhidroxi-tolueno (BHT - 100ppm). $5 \mathrm{~mL}$ de esta mezcla se considero como blanco. Los tubos se colocaron en un baño termostatado $\left(50{ }^{\circ} \mathrm{C}\right)$ y la lectura inicial a $470 \mathrm{~nm}$ fue tomada como tiempo cero. Las medidas de absorbancia se realizaron cada 15 minutos durante 120 minutos. Todas las determinaciones se llevaron a cabo en triplicado. La actividad antioxidante fue evaluada en términos del blanqueamiento del $\beta$-caroteno, usando la siguiente formula: $\mathrm{AA}(\%)=100-\left[\left(\mathrm{A}_{\mathrm{s}}^{\mathrm{o}}-\mathrm{A}^{120}{ }_{\mathrm{s}} / \mathrm{A}_{\mathrm{c}}^{\mathrm{o}}-\mathrm{A}^{120}{ }_{\mathrm{c}}\right) \mathrm{x} 100\right] ; \mathrm{A}_{\mathrm{s}}^{\mathrm{o}} \mathrm{y} \mathrm{A}^{\mathrm{o}}$ son la absorbancia de los tubos muestras y controles al tiempo cero y $\mathrm{A}^{120}{ }_{\mathrm{s}} \mathrm{y} \mathrm{A}^{120}{ }_{\mathrm{c}}$ son las absorbancias medidas en las muestras y en blanco respectivamente al final de la incubación.

\section{Ensayo de actividad atrapadora de radicales $\mathrm{O}^{\circ}$}

$\mathrm{La}$ actividad atrapadora de radicales $\mathrm{O}_{2}^{-}$fue determinada de acuerdo a Fridovich y McCord (1969), en las siguientes condiciones: NBT (nitroblue tetrazolium) $31 \mu \mathrm{M}$; xantina $240 \mu \mathrm{M}$ en tampón fosfato $(\mathrm{pH} 7,8,13$ $\mathrm{mM}$ ), en una cantidad suficiente para obtener una pendiente de incremento de la absorción espectrofotométrica (550 $\mathrm{nm}$ ) igual a $25 \times 10^{-3} \Delta \mathrm{DO} / \mathrm{min}$. Las mediciones cinéticas se realizaron en un espectrofotómetro Ultrospec III (Pharmacia-LKB, Suecia) con un programa para cinética enzimática con un tiempo de ensayo de 10 minutos. Los porcentajes de inhibición (PI) de la reducción del NBT producidos por el extracto fueron calculados a partir de la variación de su pendiente de incremento de la absorción espectrofotométrica $\left(\mathrm{S}_{\mathrm{m}}\right)$ en comparación con la de la muestra de referencia ( $\mathrm{S}_{\mathrm{r}}$, con $\mathrm{H}_{2} \mathrm{O}$ destilada) según la siguiente ecuación: $\left.\mathrm{PI}=\left[\mathrm{S}_{\mathrm{r}}-\mathrm{S}_{\mathrm{m}}\right) / \mathrm{S}_{\mathrm{r}}\right] \times 100$.

Ensayos de actividad atrapadora de radicales $\mathrm{OH}^{*}$ y unión al Fe

El método empleado (Aruoma, 1994) se realizó en las siguientes condiciones: en la mezcla reaccionante se añadió 2-desoxi- $d$-ribosa 2,8 mM; FeCl $25 \mu \mathrm{M}$; EDTA $100 \mathrm{mM}$ (en el caso del ensayo en presencia de este compuesto); $\mathrm{H}_{2} \mathrm{O}_{2}$ 2,8 mM; tampón fosfato ( $\mathrm{pH} 7,4,10 \mathrm{mM}$ ) y ácido ascórbico $100 \mu \mathrm{M}$. El extracto liofilizado de $B$. triquetrum él era resuspenso en tampón fosfato (pH 7,4, $10 \mathrm{mM}$ ) e se agregó en la mezcla reaccionante y luego de 1 hora de incubación a $37^{\circ} \mathrm{C}$, se añadió ácido tiobarbitúrico (TBA) al 1\% (v/v) en $\mathrm{NaOH} 50$ mM y ácido tricloroacético (TCA) al 2,8\% (p/v), trás lo cual se realizó una segunda incubación a $80^{\circ} \mathrm{C}$ durante 20 minutos. Finalmente, se monitoreó la absorbancia a 532 $\mathrm{nm}$ de los complejos formados con el TBA en un espectrofotómetro (Zuzi UV-4200, USA).
En ausencia de EDTA, este ensayo de degradación de 2 -desoxi- $d$-ribosa permitió evaluar la capacidad del extracto de unirse al $\mathrm{Fe}$, mientras que en presencia de EDTA, se determinó la capacidad del extracto para atrapar radicales $\mathrm{OH}^{*}$. Como controles positivos se incluyeron: dimetilsulfóxido $250 \mu \mathrm{M}$ (secuestrador de radicales $\mathrm{OH}^{*}$ ) y desferroxamina $12 \mathrm{mM}$ (agente quelante de $\mathrm{Fe}$ ), en la primera y segunda variante del ensayo, respectivamente. Los porcentajes de inhibición (PI) de la formación de sustancias reactivas al TBA (TBARS) fueron calculados a partir de la absorbancia registrada en las muestras $\left(\mathrm{A}_{\mathrm{m}}\right)$ con relación al blanco $\left(\mathrm{A}_{\mathrm{b}}\right.$, con $\mathrm{H}_{2} \mathrm{O}$ destilada) según la siguiente ecuación: $\mathrm{PI}=\left[\left(\mathrm{A}_{\mathrm{b}}-\mathrm{A}_{\mathrm{m}}\right) / \mathrm{A}_{\mathrm{b}}\right] \times 100$.

\section{Análisis estadístico}

Los resultados fueron procesados usando los programas Sigma Plot 2000 (versión 6.10 para Windows) y GraphPad Prism (versión 3.00 para Windows). Todos los experimentos se realizaron en triplicados y los resultados expresados como valores promedios \pm desviación estándar. En los ensayos de actividad antioxidante, las comparaciones se realizaron mediante ANOVA y prueba de Tukey's, con un nivel de significación estadístico de $p<0,05$. Las metodologías para determinar actividad antioxidante se compararon entre si mediante una prueba de regresión lineal para cantidades idénticas del extracto en cada punto.

\section{RESULTADOS}

\section{Composición química del alga marina Bryothamnion triquetrum}

La composición centesimal del alga $B$. triquetrum se presenta en la Tabla I. Esta alga presenta valores relativamente alto de proteínas y muy bajos de lípidos. Adicionalmente se observa valores altos de cenizas.

El contenido de compuestos lipídicos resulto bajo ( $1,3 \%$ de la composición relativa de alga seca), y al analizar la composición de ácidos grasos (Tabla II), se observa que esta alga presenta una relación de ácidos grasos saturados: insaturados relativamente alta $(2,68: 1)$ con cantidades mayoritarias de los ácidos margárico y palmítico.

El contenido de los minerales estudiados en el extracto de B. triquetrum se muestra en la Tabla III. A partir de estos resultados se puede plantear que esta alga presenta una baja cantidad de cloro en comparación con las cantidades de calcio, potasio, sodio y magnesio. Esta especie de alga roja presenta cantidades apreciables de 
TABLA I - Composición centesimal (\%) del alga marina Bryothamniom triquetrum. Las algas recién colectadas, lavadas con $\mathrm{H}_{2} \mathrm{O}$ destilada, secadas bajo sombra a temperatura ambiente y tamizadas a 32 mesh. Cada parámetro se realizo por triplicado

\begin{tabular}{cccccc}
\hline \multirow{2}{*}{ Agua } & Cenizas & \multicolumn{2}{c}{ Composición centesimal (\%) } & & \\
& Lípidos & Carbohidratos & Proteínas & Fibras \\
\hline 30 & 43 & 1,3 & 5,9 & 9,5 & 10,2 \\
\hline
\end{tabular}

TABLA II - Composición relativas (\%) de ácidos grasos saturados e insaturados del alga marina Bryothamniom triquetrum. Cada determinación se realizo por triplicado. Los ácidos grasos no identificados representan el 12,06\%

\begin{tabular}{|c|c|c|}
\hline$\overline{\text { Àcido graso }}$ & & $\begin{array}{l}\text { Composición } \\
\text { relativa }(\%)\end{array}$ \\
\hline Hendecanoico & C 11:0 & 0,58 \\
\hline Laurico & C 12:0 & 0,385 \\
\hline Miristico & C $14: 0$ & 1,27 \\
\hline Pentadecanoico & C 15:0 & 0,49 \\
\hline Palmitico & C 16:0 & 18,47 \\
\hline Palmitoleico & C $16: 1$ & 5,76 \\
\hline Margarico & C 17:0 & 41,24 \\
\hline Estearico & C 18:0 & 1,59 \\
\hline Oleico & C $18: 1$ & 7,99 \\
\hline Linoleico & C $18: 2 n-6$ & 1,73 \\
\hline$\gamma$-Linolénico & C $18: 3 n-6$ & 0,74 \\
\hline Araquidonico & C $20: 4 n-6$ & 3,51 \\
\hline Docosapentaenoico & C $20: 5 n-3$ & 3,73 \\
\hline Total & & 87,94 \\
\hline
\end{tabular}

bromo. Resulta importante señalar que las cantidades presentes de metales tóxicos como el mercurio y el talio son muy bajas y/o no detectables mientras que se observan altas cantidades de plomo. Algunos minerales pueden modular el balance oxidativo celular ( $\mathrm{Se}, \mathrm{Zn}$ e Mg), y de ahí que sea necesario conocer el contenido de ellos en el extracto. Las cantidades de los metales que pueden tener efectos pro-oxidantes como el hierro, cobre, cadmio y niquel son bajos. El contenido de otros minerales como cobalto, cromo y mercurio que también pueden promover el estrés oxidativo, son tan bajos que no puede ser detectado con la metodología experimental empleada. Por otra parte, el extracto contiene cantidades apreciables de selenio que es un mineral para el que se han referido funciones antioxidantes en el organismo.

Entre otros de los compuestos del alga con funciones antioxidantes específicas se encuentra el ácido ascórbico y los carotenoides, cuyos contenidos son de $0,4 \pm 0,01$ y
TABLA III - Composición de minerales del extracto acuoso liofilizado de B.triquetrum, expresado en $\mathrm{mg} / \mathrm{kg}$ de extracto liofilizado. Todas las determinaciones, realizadas por espectrometría de absorción átomica, se repitieron 5 veces por duplicado

\begin{tabular}{lc}
\hline Mineral & Contenido $(\mathbf{m g} / \mathbf{k g})($ media $\pm \mathbf{D S})$ \\
\hline $\mathrm{Na}$ & $31250 \pm 40$ \\
$\mathrm{Mg}$ & $2900 \pm 30$ \\
$\mathrm{Cl}$ & $5,5 \pm 0,05$ \\
$\mathrm{~K}$ & $63900 \pm 200$ \\
$\mathrm{Ca}$ & $6600 \pm 120$ \\
$\mathrm{Zn}$ & $18 \pm 1,5$ \\
$\mathrm{Br}$ & $15 \pm 0,5$ \\
$\mathrm{~Pb}$ & $7,5 \pm 0,1$ \\
$\mathrm{Tl}$ & 000 \\
$\mathrm{Fe}$ & $200 \pm 50$ \\
$\mathrm{Cu}$ & $20 \pm 2$ \\
$\mathrm{Cd}$ & $0,20 \pm 0,08$ \\
$\mathrm{Ni}$ & $10 \pm 5$ \\
$\mathrm{Co}$ & 000 \\
$\mathrm{Cr}$ & 000 \\
$\mathrm{Hg}$ & 000 \\
$\mathrm{Se}$ & $100 \pm 9$ \\
\hline
\end{tabular}

$0,04 \pm 0,005 \mathrm{mg} / \mathrm{g}$ (muestra liofilizada) respectivamente, valores que pueden ser considerados bajos.

\section{Actividad antioxidante}

Actividad antioxidante en el ensayo de atrapamiento de radicales $D P P H^{\circ}$

Los resultados de la actividad atrapadora de radicales $\mathrm{DPPH}^{\circ}$ se muestran en la Figura 1. Como se puede apreciar el extracto de B. triquetrum es efectivo en el atrapamiento de estos radicales sólo a altas concentraciones, aunque presenta una relación directa entre las concentraciones del extracto y la actividad biológica, con un valor de $\mathrm{CI}_{50}$ en este modelo de 4,66 mg (muestra liofilizada). 


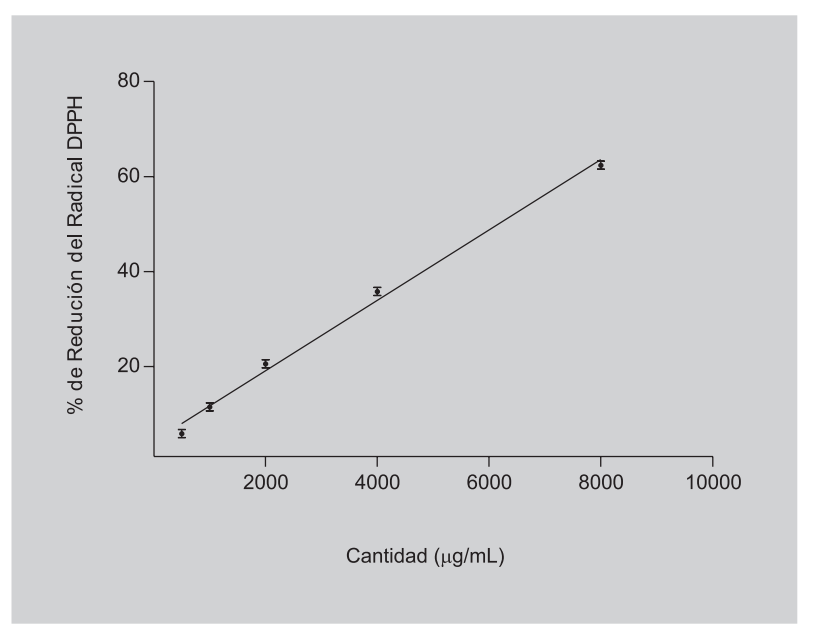

FIGURA 1 - Actividad antioxidante del extracto acuoso de B.triquetrum contendo de 0,5 a $8 \times 10^{3} \mu \mathrm{g} / \mathrm{mL}$ mediante el ensayo de atrapamiento de radicales DPPH. Los resultados están expresados como $\mathrm{x} \pm \mathrm{DS}$ en función del blanqueamiento del DPPH. Cada determinación se realizo por triplicado.

Actividad antioxidante por el ensayo del $\beta$-Caroteno-ácido linoléico

La actividad antioxidante determinada por el ensayo del $\beta$-Caroteno-ácido linoléico se muestra en la Figura 2. Cuando se compararon los resultados de actividad antioxidante en el sistema $\beta$-caroteno/linoléico con el ensayo del DPPH, para la concentración de $4 \mathrm{mg}$ de extracto, se observo un valor de inhibición de la oxidación mas bajo (12\%).

Los resultados de actividad antioxidante por ensayo del $\beta$-Caroteno-ácido linoléico y de la actividad atrapadora de radicales DPPH'se relacionaron estadísticamente (Figura 3) y se obtuvo una correlación lineal satisfactoria entre estos dos parámetros $\left(\mathrm{r}^{2}=0,9108\right)$.

\section{Evaluación de la capacidad atrapadora de radicales libre} y unión al Fe

La capacidad del extracto de B.triquetrum para interaccionar con los radicales $\mathrm{O}_{2}^{-}$- fue medida a través de su efecto inhibidor sobre la reducción del NBT provocada por estos radicales (Tabla IV). Se aprecia que el extracto de $B$. triquetrum presenta capacidad para reaccionar directamente con los radicales $\mathrm{O}_{2}{ }^{-}$de un modo dependiente de su concentración. El porcentaje de inhibición de la reducción del NBT mantiene una relación sigmoidal con el logaritmo de la concentración y el valor determinado de $\mathrm{CI}_{50}$ es $0,36 \mathrm{mg} / \mathrm{mL}$. No se puede determinar la constante de velocidad de la reacción con los radicales $\mathrm{O}_{2}{ }^{--}$por tratarse de un
TABLA IV - Actividad atrapadora de radicales $\mathrm{O}_{2}{ }^{-}$- del extracto acuoso liofilizado de $B$. triquetrum. Los experimentos se repitieron 2 veces con 5 réplicas en cada caso

\begin{tabular}{lcc}
\hline Muestra & $\begin{array}{c}\text { [extracto de } \text { B. triquetrum] } \\
(\mathbf{m g} / \mathbf{m L})\end{array}$ & PI (\%) \\
\hline B.triquetrum & 0,1 & 9 \\
& 0,2 & 18 \\
& 0,3 & 36 \\
& 0,5 & 55 \\
& 0,8 & 68 \\
& 1,9 & 81 \\
& 2,8 & 90 \\
Blanco & 4,7 & 99 \\
\hline
\end{tabular}

PI representa el porcentaje de la reducción del NBT (nitroblue tetrazolium). Se empleó ANOVA y test de Tukey para la comparación de las muestras, con nivel de significación de $\mathrm{p}<0,05$

extracto crudo, que es una mezcla de compuestos con diversa naturaleza química.

Los resultados del ensayo en el que se evaluó la habilidad del extracto para impedir la oxidación de la 2desoxi- $d$-ribosa mediada por radicales $\mathrm{OH}^{*}$, se presentan en la Tabla V. Este ensayo se realiza en dos condiciones experimentales diferentes: en presencia y en ausencia de EDTA, que permiten estudiar la capacidad para atrapar radicales $\mathrm{OH}^{*}$ y para unir Fe respectivamente. En ambos casos, el extracto muestra un efecto protector dependiente de la concentración y los valores de $\mathrm{CI}_{50}$ determinados son $0,37 \mathrm{mg} / \mathrm{mL}$ (en presencia de EDTA) y $2,11 \mathrm{mg} / \mathrm{mL}$ (en ausencia de EDTA).

\section{DISCUSIÓN}

Las algas marinas, consideradas como un alimento tradicional en algunos países, especialmente en los asiáti$\cos$, han sido vinculadas a la prevención y/o tratamiento de diferentes enfermedades relacionadas con el estrés oxidativo, aspecto que ha servido de pauta para las investigaciones de sus propiedades antioxidantes (Funahashi et al., 2001; Jiménez-Escrig, Sánchez-Muñiz, 2000; Yuan, Walsh, 2006).

En el contexto de la posible utilización del alga marina B.triquetrum como alimento funcional $\mathrm{y} / \mathrm{o}$ fitofármaco con propiedades antioxidantes, se hace necesario analizar su composición centesimal (Tabla I). En las algas, de acuerdo a sus necesidades metabólicas, se encuentran cantidades bajas de lípidos y relativamente al- 
TABLA V - Efecto protector del extracto acuoso liofilizado de B.triquetrum sobre la degradación oxidativa de la 2-desoxi$d$-ribosa mediada por radicales $\mathrm{OH}^{*}$

\begin{tabular}{llcc}
\hline Ensayo & Muestra & [extracto de B.triquetrum] (mg/mL) & PI (\%) \\
\hline \multirow{3}{*}{ En presencia de EDTA } & Blanco & 0 & - \\
\cline { 2 - 4 } & B. triquetrum & 0,1 & 3 \\
& & 0,2 & 17 \\
& & 0,3 & 39 \\
& & 0,5 & 45 \\
& & 0,8 & 59 \\
& & 1,5 & 73 \\
& & 2,5 & 80 \\
En ausencia de EDTA & 3,5 & 83 \\
& Blanco & 4,5 & 91 \\
& B. triquetrum & 5,0 & 95 \\
& & 0 & - \\
\cline { 2 - 3 } & & 0,75 & 18 \\
& & 1,00 & 27 \\
& & 1,25 & 34 \\
& & 1,50 & 42 \\
& & 2,00 & 52 \\
& & 2,50 & 63 \\
& & 3,00 & 75 \\
\end{tabular}

PI representa el porcentaje de inhibición de la oxidación de la 2-desoxi- $d$-ribosa. Los experimentos se repitieron 2 veces con 5 réplicas en cada caso. Se empleó ANOVA y test de Tukey para la comparación de las muestras, con nivel de significación de $\mathrm{p}<0,05$. Se incluyeron dos controles: con dimetilsulfóxido $250 \mu \mathrm{M}$ (en presencia de EDTA) y desferroxamina $12 \mathrm{mM}$ (en ausencia de EDTA)

tas de proteínas (Jiménez-Escrig, Goñi, 1999), criterios confirmados en este trabajo. Carrillo et al., (2002) encontraron valores proteicos para diferentes especies de algas rojas similares al encontrado para $B$. triquetrum. Huang y Wang (2004) determinaron para el alga Plocamium telfairiae una relación de ácidos saturados: insaturados alta, debido fundamentalmente al ácido palmitico, resultados similares encontrados en el alga $B$. triquetrum, que presenta concentraciones de ácido margárico y palmítico mayores que del resto de los ácidos grasos examinados (Tabla II). Se ha considerado que los ácidos grasos insaturados de las algas pudieran representar una fuente de antioxidantes naturales (Huang, Wang, 2004), pero si consideramos el bajo contenido de estos compuestos de esta alga, su actividad antioxidante debiera explicarse por la presencia de otras entidades.

En general, las algas marinas presentan altos contenidos de compuestos inorgánicos (Felicio-Fernandes, Laranjeira, 2000). En esta investigación el contenido de minerales del extracto resultó elevado lo que pudiera explicar el valor elevado de cenizas. Vidal et al. (2001) aislando ácidos fenólicos de extractos de esta alga encontraron en algunas fracciones cantidades elevadas de compuestos inorgánicos. También Risso et al. (2003) reportaron valores elevados de cenizas en el alga Monostroma undulatum.

Diferentes autores han referido la presencia cantidades significativas de compuestos bromados en las algas marinas rojas (Whitfield et al., 1999), que de hecho han sido consideradas como fuentes de estos compuestos, entonces los niveles de bromo encontrados en esta alga se corresponden con los hallazgos anteriores.

En estudios previos se relacionó la actividad antioxidante del extracto acuoso de $B$. triquetrum con su contenido de ácidos $p$-cumárico, $t$-cinámico y ferúlico (Vidal et al., 2001, Fallarero et al., 2006). Adicionalmente Fallarero et al. (2006) demostraron en estudios de cultivos de células neuronales GT1-7 que estos ácidos administrados separadamente y/o en conjunto no explicaban to- 
talmente la actividad antioxidante de esta alga. Otros metabolitos con función antioxidante, que en alguna medida pudieran influir en esta propiedad son los carotenos y el ácido ascorbico, también presentes en este extracto. En algunas especies de algas marinas se ha comprobado que la actividad antioxidante se encuentra en función de estos compuestos (Yan et al.,1999). Por otro lado la actividad de estos compuestos polifenólicos pudiera ser favorecida por la presencia del ácido ascórbico en el extracto provocando una acción antioxidante potenciada, tal y como ha sido referido por otros autores en modelos experimentales in vitro (Vieira et al., 1998).

Las algas contienen elementos trazas necesarios para su metabolismo celular aunque adicionalmente se observan cantidades excesivas debido a la absorción de estos minerales del medio ambiente (Collen et al., 2003). Por ello, el uso de algas como fuentes alimenticias podría conllevar la ingestión de cantidades apreciables de estos elementos, algunos de ellos nocivos. Es interesante señalar que el alga $B$. triquetrum no contiene elementos tóxicos como el mercurio y el talio. En el estrés oxidativo los elementos trazas, en algunos casos son fundamentalmente pro-oxidantes como el hierro (a través de la reacción de Fenton) mientras otros como el cobre y zinc son indispensables para la actividad de enzimas antioxidantes como la superóxido-dismutasa (SOD) y como inductores de la expresión de metalotioneínas, consideradas proteínas detoxificantes (Klotz et al., 2003). El selenio es considerado el mineral traza mas importante como antioxidante, debido a su papel indispensable en la composición de algunas enzimas (Klotz et al., 2003). Es importante señalar que el contenido de elementos trazas de esta alga que pudieran actuar como pro-oxidantes como el hierro, niquel y cobre se encuentran en niveles bajos en los que no resultan especialmente tóxicos mientras que otros como el selenio, aunque con un contenido bajo, pudieran coadyuvar a la propiedad antioxidante del extracto (Tabla III).

Las propiedades nutricionales $\mathrm{y} / \mathrm{o}$ farmacológicas de un extracto vegetal, en la mayoría de los casos se encuentran en función de un conjunto de moléculas, donde unas pocas son mayoritarias, sin embargo de alguna manera, el resto de las moléculas mimetizan esos efectos (Tucker, 2003). Aunque en esta alga juegan un papel importante los compuestos polifenólicos como los ácidos cinámicos como fue demostrado en trabajos previos de nuestro grupo de investigación, todo evidencia que la presencia de otros tipos de moléculas además de los compuestos fenólicos y/o un posible efecto sinérgico entre todos estos compuestos, en última instancia explicarían esta propiedad. El extracto puede disminuir la generación de radicales libres, posiblemente por la combinación de sus habilidades en la captura de los radicales libres, la unión al Fe y el efecto sinérgico con moléculas antioxidantes endógenas.

En investigaciones previas, se demostró que el alga marina B.triquetrum presenta actividad neuroprotectora relacionada directamente con su actividad antioxidante (Vidal et al., 2001; Fallarero et al., 2002, 2003, 2006), no obstante aún se trabaja en la total comprensión de los mecanismos moleculares que sustentan estas propiedades neuroprotectoras.

La capacidad atrapadora de radicales libres mediante el ensayo del DPPH, evidencia que el extracto presenta una actividad antioxidante aunque baja, comparable a otros extractos. En una investigacion previa (Fallarero et al.,2006) se estudio la actividad atrapadora de radicales DPPH con una única dosis con resultados similares. La CI $_{50}$ (Figura 1) obtenida en este modelo fue similar al referido para el extracto metanólico antioxidante del alga parda Undaria pinnatifida (Han et al.,2002). Adicionalmente se investigó esta propiedad con el ensayo del Sistema $\beta$-Caroteno-linoléico (Figura 2), basado en la capacidad de un antioxidante de proteger la lipoperoxidación mediante el atrapamiento de radicales libres tanto en la fase de iniciación como de propagación, resultados que evidencian una débil actividad atrapadora de radicales libres del extracto del alga B.triquetrum. Al analizar estadísticamente la correlación de estas dos metodologías (Figura 3 ) se encontró un coeficiente de correlación satisfactoria $\left(\mathrm{r}^{2}=0,912\right)$, y esto permite avalar el criterio de que el mecanismo de acción antioxidante de esta alga en cierta medida está relacionado con la capacidad atrapadora de radicales libres. Si consideramos

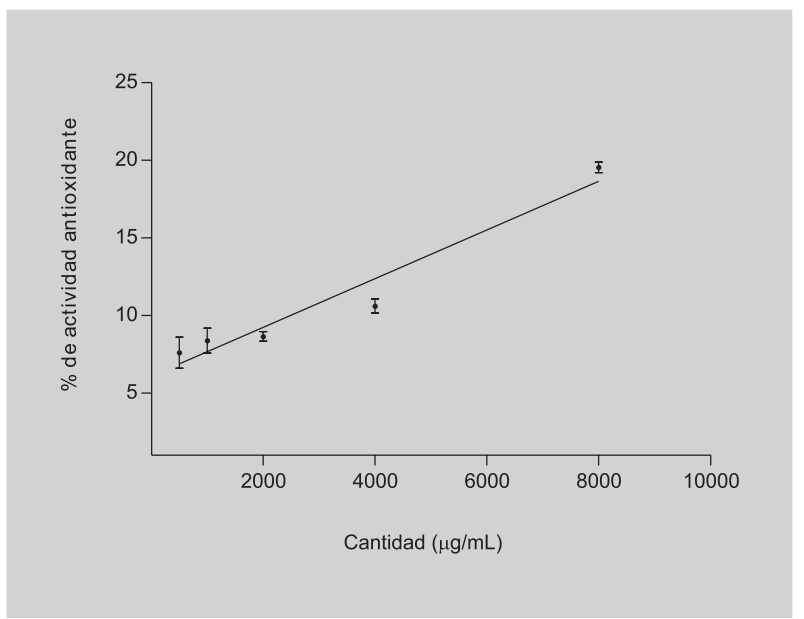

FIGURA 2 - Actividad antioxidante del extracto acuoso de $B$. triquetrum contendo de 0,5 a $8 \times 10^{3} \mu \mathrm{g} / \mathrm{mL}$ mediante el ensayo de sistema $\beta$-caroteno-ácido linoleico. Los resultados están expresados como $\mathrm{x} \pm \mathrm{DS}$ en función del blanqueamiento del $\beta$-caroteno. Cada determinación se realizo por triplicado. 
trabajos previos (Vidal et al., 2001; Fallarero et al., 2006) donde se relacionan la actividad antioxidante de esta alga con la presencia de polifenoles, entonces ademas se debe considerar que los compuestos polifenolicos pueden actuar como antioxidantes no solo por un mecanismo de atrapamiento de radicales libres (Rice-Evans et al., 1995; Yeh, Yen, 2006). Por otra parte, la actividad antioxidante pudiera ser explicada por un conjunto de mecansimos. Kang et al. (2005) encontraron que el alga roja Callophyllis japonica presentaba actividad atrapadora de radicales DPPH; inhibía la peroxidación lipídica y además incrementaba la actividad de algunas enzimas antioxidantes.

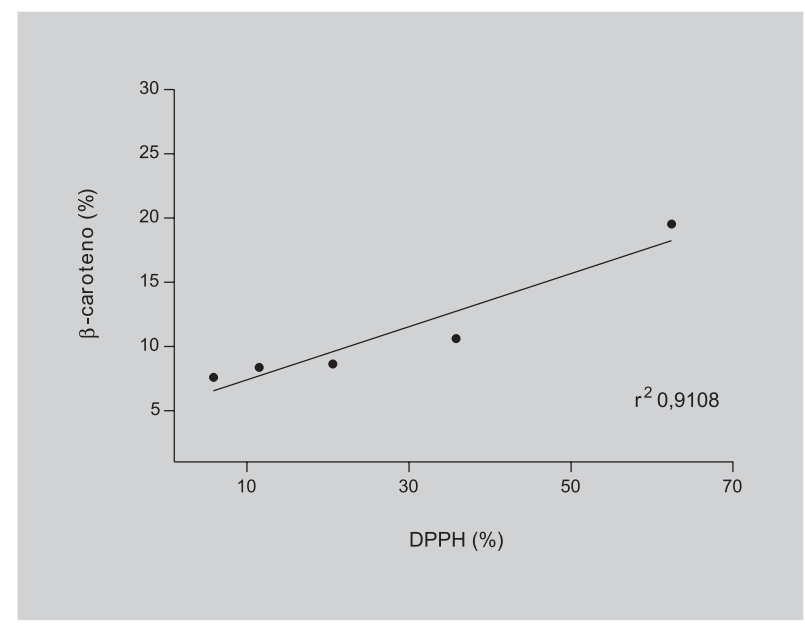

FIGURA 3 - Estudio de correlación lineal de la actividad antioxidante del extracto acuoso de $B$. triquetrum mediante los ensayos de sistema $\beta$-caroteno-ácido linoléico y de atrapamiento de radicales DPPH. En cada punto se emplearon cantidades idénticas del extracto liofilizado $(0,5$; $1 ; 2 ; 4$ y $8 \mathrm{mg}$ ).

La capacidad del extracto de $B$. triquetrum para interactuar con los radicales $\mathrm{O}_{2}{ }^{-}$fue medida a través de su efecto inhibidor sobre la reducción del NBT provocada por estos radicales (Tabla IV). Los radicales $\mathrm{O}_{2}^{--}$pueden mediar directamente el daño a proteínas y estimular además la generación de otras entidades aún más reactivas como el radical $\mathrm{OH}^{*}$, implicados en el daño a las biomoléculas, como los lípidos, que ocurre ante situaciones de estrés oxidativo (Halliwell et al., 1995), de manera que la captura de estos radicales estaría en función de moléculas no enzimáticas contenidas en el extracto de B.triquetrum capaces de atrapar radicales $\mathrm{O}_{2}^{-}$e impedir la oxidación de la 2desoxi- $d$-ribosa mediada por radicales $\mathrm{OH}^{*}$ en ausencia de EDTA (Scott et al., 1993).

En la primera variante del experimento de la actividad atrapadora de radicales $\mathrm{OH}^{\circ}$, se muestra la habilidad del extracto para competir con la 2-desoxi- $d$ ribosa en la reacción con esos radicales que son generados por la reacción de Fenton que tiene lugar en la mezcla del ácido ascórbico, $\mathrm{H}_{2} \mathrm{O}_{2}$ y $\mathrm{Fe}^{3+}$-EDTA (Halliwell et al., 1995). El valor de $\mathrm{CI}_{50}$ para atrapar radicales $\mathrm{OH}$ obtenido es menor que los referidos para diferentes extractos vegetales antioxidantes (Matsuda et al., 1998; Babu et al., 2001; Lin et al., 1995), entonces, el extracto de B.triquetrum muestra una mayor efectividad como atrapador de estos radicales en comparación con dichos extractos (Tabla V). Cuando los iones $\mathrm{Fe}^{3+}$ son añadidos a la mezcla de reacción (en ausencia de EDTA), algunos de ellos pueden unirse a la 2 -desoxi- $d$-ribosa y así enlazados pueden participar en la reacción de Fenton, de modo que cada $\mathrm{OH}^{*}$ formado inmediatamente atacará el azúcar y no se liberará al medio (Aruoma, 1994; Halliwell et al., 1995). La actividad antioxidante medida en este ensayo muestra la capacidad de los componentes del extracto de B.triquetrum para competir con la 2-desoxi$d$-ribosa en la unión al Fe. $\mathrm{La} \mathrm{CI}_{50}$ en la variante del ensayo que permite detectar la capacidad de unión a los radicales $\mathrm{OH}^{*}$ (con EDTA) fue aproximadamente 6 veces menor que la calculada para la unión al Fe. El valor de $\mathrm{CI}_{50}$ obtenido en el primer caso es similar al referido para un extracto de Ginkgo biloba (Guidetti et al., 2001), resultados que sugieren la prersencia en el extracto de moléculas que pueden reaccionar directamente con el radical formado y así evitar la reacción de éste con la 2-desoxi$d$-ribosa. Los resultados obtenidos en la segunda variante del ensayo ( $\sin$ EDTA) demuestran que, además, el extracto tiene la habilidad de interferir la generación "sitio específica" de $\mathrm{OH}^{*}$ catalizada por los iones Fe enlazados a las moléculas del azúcar. Se sugiere que esta actividad del extracto se relaciona con la combinación de dos factores: i) la habilidad para evitar la unión de la 2-desoxi$d$-ribosa al Fe (o de abstraerlo en caso que haya ocurrido el enlazamiento) y ii) la habilidad para "inactivar" los iones Fe que son abstraídos e impedir su participación en la reacción de Fenton.

\section{CONCLUSIONES}

En conclusión se puede señalar que el alga marina roja Bryothamnion triquetrum (S.G.Gmelim) Howe presenta una composición centesimal caracterizada por cantidades bajas de lípidos y proteínas, valores altos de carbohidratos y de minerales, que podrían explicar el contenido elevado de cenizas. Aunque en trabajos precedentes se identificaron los ácidos cinámicos y fenólicos entre los responsables de esta propiedad, es necesario considerar un efecto sumatorio y/o sinérgico de otros componentes antioxidantes como 
carotenos, ácido ascórbico y selenio. La actividad antioxidante que a su vez pudiera ser explicada en parte por la capacidad atrapadora de radicales libres parece estar más vinculada a un mecanismo de dismutación de radicales $\mathrm{O}_{2}{ }^{\cdot}$ , inactivación de radicales $\mathrm{OH}^{*}$ y a la quelación de Fe. Los resultados de este trabajo avalan las potencialidades de esta alga marina roja como fuente de antioxidantes naturales con perspectivas de aplicación como fitofármaco y/o suplemento dietético funcional.

\section{ABSTRACT}

\section{Chemical composition and antioxidant activity of the red marine algae Bryothamnion triquetrum (S.G.Gmelin) Howe}

An increasing interest has been growing during the past years for the search of natural origin antioxidants, particularly those from marine algae. In this context, the main objective of current research was to evaluate the chemical composition and some antioxidant properties of the aqueous extract of the seaweed Bryothamnion triquetrum. The extracts contains: Proteins (9.5\%), Lipids (1.3\%), Carbohydrates (5.9\%), Fibers (10.2\%) and Ashes (43\%). In current approach, the following results were obtained for the different procedures assessed: DPPH radicals scavenging (38\% for $4 \mathrm{mg}$ of lyophilized); $\beta$ Carotene-Linoleic assay (12\% for $4 \mathrm{mg}$ of lyophilized); $\mathrm{O}_{2}{ }^{-}$radicals scavenging $\left(\mathrm{IC}_{50} 0.36 \mathrm{mg} / \mathrm{mL}\right) ; \mathrm{OH}^{*}$ radicals scavenging $\left(I C_{50} 2.11 \mathrm{mg} / \mathrm{mL}\right)$ and iron quelation ability $\left(I C_{50} 0.37 \mathrm{mg} / \mathrm{mL}\right)$. Thus, antioxidant properties of this natural product seem to be related to its ability to scavenge free species. In previous reports of our group, cinnamic and phenolic acids were proposed as at least partially responsible for the antioxidant properties of the extract, but the necessity for the presence of other components was also shown. Then, the antioxidant properties of the extract could be envisioned as the result of the additive and/or synergic effect between phenolic constituents and the other antioxidant components, such as minerals, carotenes and ascorbic acid.

UNITERMS: Seaweeds. Bryothamnion triquetrum. Chemical composition. Antioxidant activity.

\section{AGRADECIMIENTOS}

Quisiéramos expresar nuestro agradecimiento a $\mathrm{CNPq}$ por el otorgamiento del proyecto 300064/2004-7 “Alimentos funcionales: Las algas marinas y las microalgas como fuentes de antioxidantes naturales y de alimentos alternativos".

\section{REFERENCIAS}

ASSOCIATION OFFICIALOFANALYTICALCHEMISTS. Official methods of analysis of the AOAC.Arlington:AOAC Internartional, 1990. p.1048.

ASSOCIATION OFFICIALOFANALYTICALCHEMISTS. Official Methods of Analysis of the AOAC. Arlington: AOAC International, 1995. cap IV, p.2;4;7;17;18.

ASSOCIATION OFFICIAL OF ANALYTICAL CHEMISTS. Official Methods of Analysis of the AOAC.Arlington: AOAC International, 2002. cap. 41 p.20-26.

ARECES, A. Biotecnología de agarofitas del género Bryothamnion kützing. Habana, 1995. p.30-64. [Tesis Doctorado en Ciencias Biológicas. Instituto de Oceanología. Universidad de La Haban, Cuba].

ARUOMA, O.I.; BAHORUN, T.; JEN, L.S. Neuroprotection by bioactive components in medicinal and food plant extracts. Mutat. Res., v.544, n.23, p.203-215, 2003.

ARUOMA, O.I. Deoxyribose assay for detecting hydroxyl radicals. Methods Enzymol., v. 233, p.57-66, 1994.

BABU, B.H.; SHYLESH, B.S.; PADIKKALA, J. Antioxidant and hepatoprotective effect of Acanthus ilicifolius. Fitoterapia, v.72, p. 272-277, 2001.

BURRITT, D.; LARKINDALE, J.; HURD, C.L. Antioxidant metabolism in the intertidal red seaweed Stictosiphonia arbuscula following desiccation. Planta, v.215, n.5, p. 829-838, 2002.

COLLEN, J.; PINTO, E.; PEDERSEN, M.; COLEPICOLO, P. Induction of oxidative stress in the red macroalga Gracilaria tenuistipitata by pollutant metals. Arch. Environ. Contam. Toxicol., v.45, n.3, p.337-342, 2003.

CARRILLO, S.; CASAS, M.; RAMOS, F.; PEREZ-GIL, F.; SANCHEZ, I. Marine algae of Baja California Sur, Mexico: nutritional value. Arch. Latinoam. Nutr., v.52, n. 4, p.400-405, 2002.

FALLARERO, A.; LOIKKANEN, J.J.; MÄNNISTÖ, P.T.; CASTAÑEDA, O.; VIDAL, A. Effects of aqueous extracts of Halimeda incrassata (Ellis) Lamouroux and Bryothamnion triquetrum (S.G.Gmelim) Howe on hydrogen peroxide and methyl mercury-induced oxidative stress in GT1-7 mouse hypothalamic immortalized cells. Phytomedicine, v.10, n.1 p. 39-47, 2003. 
FALLARERO, A.; VIDAL, A., VUORELA, P. Protective effect of the aqueous extract of Bryothamnion triquetrum against aglycemia and chemical hypoxia-induced damage in GT1-7 immortalized mice hypothalamic cell line. Eur. $J$. Pharm. Sci., v. 17, suppl. 1, S42, 2002.

FALLARERO, A.; PELTOKETO, A.; LOIKKANEN, J.; TAMMELA, P.; VIDAL, A.; VUORELA, P. Effects of aqueous extracts of Bryothamnion triquetrum on chemical hypoxia and aglycemia-induced damage in GT1-7 mouse hypothalamic immortalized cells. Phytomedicine. v.13,n.4, p.240-245, 2006.

FELICIO-FERNANDES, G.; LARANJEIRA, C.M. Calcium phosphate biomaterials from marine algae. Hydrotermical synthesis and characterisation. Quim. Nova, v.23, n.4, p.441-446, 2000.

FOLCH, J.; LEES, M.; STANLEY, G.H.S. A simple method for the isolation and purification of total lipides from animal tissues. J. Biol. Chem., v.226, p.497-509, 1957.

FRIDOVICH, I.; MC CORD, J.M. Superoxide dismutase. An enzymic function for erythrocuprein (hemocuprein). $J$. Biol. Chem., v. 244, p.6049-6055, 1969.

FUNAHASHI, H.; IMAI, T.; MASE, T.; SEKIYA, M.; YOKOI, K.; HAYASHI, H.; SHIBATA, A.; HAYASHI, T.; NISHIKAWA, M.; SUDA, N.; HIBI, Y.; MIZUNO, Y.; TSUKAMURA, K.; HAYAKAWA, A.; TANUMA, S. Seaweed prevents breast cancer? Jpn. J. Cancer Res., v.92, n.5, p.483-487, 2001.

GOUPY, P.; HUGUES, M.; BOIVIN, P.; AMIOT, M.J. Antioxidant composition and activity of barley (Hordeum vulgare) and malt extracts and of isolated phenolic compounds. J. Sci. Food Agric., v.79, p.1625-1634, 1999.

GUIDETTI, C.; PARACCHINI, S.; LUCCHINI, S.; CAMBIERI, M.; MARZATICO, F. Prevention of neuronal cell damage induced by oxidative stress in vitro: effect of different Ginkgo biloba extracts. J. Pharm. Phamacol., v.53, p.387-392, 2001.

HALLIWELL, B.; AESCHBACHT, R.; LOLIGERT, J.; ARUOMA, O.I. The characterization of Antioxidants. Food Chem. Toxic., v.33, n. 7, p. 601-617, 1995.

HAN, J.; XING, D.; SUN, H.; LU, H.; LI, M.; DU, L. Comparison of flavonoids, terpenoids and their natural complex in Ginkgo biloba on antioxidant effect. Zhongguo Yaolixue Tongbao, v.18, n.1, p. 115-117, 2002.
HARTMAN, L.; LAGO, R.C.A. Rapid preparation of fatty acid methyl esters from lipids. Lab. Pract., v.22, p. 475477, 1973.

HUANG, L.H.; WANG, B.G. Antioxidant capacity and lipophilic content of seaweeds collected from the Qingdao coastline. J. Agric. Food Chem., v.58, p.4993-4997, 2004.

JIMÉNEZ-ESCRIG, A.; GOÑI, I. Nutritional evaluation and physiological effects of edible seaweeds. Arch. Latinoam. Nutr., v.49, n.2, p.114-120, 1999.

JIMÉNEZ-ESCRIG, A.; JIMÉNEZ-JIMÉNEZ, I.; PULIDO, R.; SAURA-CALIXTO, F. Antioxidant activity of fresh and processed edible seaweeds. J. Sci. Food Agric., v.81, p.530-534, 2001.

JIMÉNEZ-ESCRIG, A.; SÁNCHEZ-MUÑIZ, F.J. Dietary fiber from edible seaweeds: chemical structure, physicochemical properties and effects on cholesterol metabolism. Nutr.Res., v.20, n.4, p. 595-598, 2000.

KLOTZ, L.O.; KRONCKE, K.D.; BUCHCZYK, D.P.; SEIS, H. Role of cooper, zinc, selenium and tellurium in the cellular defense against oxidative and nitrosative stress. J. Nutr., v. 133, p. 1448S-1451S, 2003

KUMPULAINEN, J.; RAITTILA, A.M.; LEHTO, J.; KOIVISTOINEN, P. Electrothermal atomic absorption spectrometric determination of selenium in foods and diets. J.Assoc. Off. Anal. Chem., v.66, n.5, p.1129-1135, 1983.

LIM, S.N.; CHEUNG, P.C.; OOI, V.E.; ANG, P.O. Evaluation of antioxidative activity of extracts from a brown seaweed, Sargassum siliquastrum. J. Agric. Food Chem., v.50, n.3, p.3862-3866, 2002.

LIN, J.M.; LIN, C.C.; CHEN, M.F.; UJIIE, T.; TAKADA, A. Radical scavenger and antihepatotoxic activity of Ganoderma formosanum, Ganoderma lucidum and Ganoderma neo-japonicum. J. Ethnopharmacol., v.47, p.33-41, 1995.

LINARES, A.F.; LOIKKANEN, J.; JORGE, M.F.; SORIA, R.B.; NOVOA, A.V. Antioxidant and neuroprotective activity of the extract from the seaweed, Halimeda incrassata (Ellis) Lamouroux, against in vitro and in vivo toxicity induced by methyl-mercury. Vet. Hum. Toxicol., v.46, n.1, p.1-5, 2004 
MATSUDA, H.; ISHIKADO, A.; NISHIDA, N.; NINOMIYA, K.; FUJIWARA, H.; KOBAYASHI, Y.; YOSHIKAWA, M. Hepatoprotective, superoxide scavenging and antioxidative activities of aromatic constituents from the bark of Betula platyphylla var.japonica. Bioorg. Med. Chem. Lett., v.8, p.2939-2944, 1998.

MILLER, H.E.A. A simplified method for the evaluation of antioxidants. J. Am. Oil Chem. Soc., v.48, p. 91, 1971.

NATELSON, S. Techniques of clinical chemistry. Springfeld: Charles G. Thomas, 1964. p.162-165.

RAGHAVENDRAN, H.R. ; SATHIVEL, A. ; DEVAKI, T. Protective effect of Sargassum polycystum (brown alga) against acetaminophen-induced lipid peroxidation in rats. Phytother. Res., v.19, n.2, p. 113-115, 2005.

RICE-EVANS, C.A.; MILLER, N.J.; BOLWELL, P.G.; BRAMLEY, P.M.; PRIDHAM, J.B. The relative antioxidant activities of plant-derived polyphenolic flavonoids. Free Radical Res., v.22, n.4, p.375-383, 1995.

RISSO, S.; ESCUDERO, C.; ESTEVAO, S.; DE PORTELA, M.L.; FAJARDO, M.A. Chemical composition and seasonal fluctuations of the edible green seaweed, Monostroma undulatum, Wittrock, from the Southern Argentina coast. Arch. Latinoam. Nutr., v.53, n.3, p. 306-311, 2003.

SAARI, E.; PAASO, A. Mineral element composition of finnish foods II: analytic methods. Acta Agric. Scand., v.22, p. 15-25, 1980.

SCOTT, B.C.; BUTLER, J.; HALLIWELL, B.; ARUOMA, O.I. Evaluation of the antioxidant actions of ferulic acid and catechins. Free Radical Res. Commun., v.19, n.4, p. 241-253, 1993.

TUCKER, G. Nutritional enhancement of plants. Curr. Opin. Biotechnol., v.14, p.221-225, 2003.

VIDAL,A.; FALLARERO, A.; VUORELA, P. Toxicological evaluations of plants extracts: Is it necessary? In: GOVIL, N. (Ed.). Recent progress in medicinal plants. Houston: Studium Press, 2005. v. 10, p. 1-14. (Phytotherapeutics Review series).
VIDAL,A.;MOTIDOME, M.; TANAE, M.; FALLARERO, A.; BRANDAO, L.; MANCINI, J.; LAPA, A.J. Actividad antioxidante y ácidos fenólicos del alga marina Bryothamnion triquetrum. Rev. Bras. Cienc. Farm., v. 37, n. 3, p. 373-382, 2001.

VIEIRA, O.; ESCARGUEIL-BLANC, I.; MEILHAC, O.; BASILE, J.P.; LARANJINHA, J.; ALMEIDA, L.; SALVAYRE, R.; NĖGRE-SALVAYRE, A. Effect of dietary phenolic compounds on apoptosis of human cultured endothelial cells induced by oxidized LDL. Br. J. Pharmacol., v.123, p. 565-573, 1998.

WHITFIELD, F.B.; FLODIN, C.; HELIDONIOTIS, F.; SHAW, K.J.; SVOIRONOS, D. Distribution of bromophenols in species of marin algae from eastern, Australia.J. Agric. Food Chem., v.47, p.2367-2373, 1999.

YAN, X.; CHUDA, Y.; SUZUKI, M.; NAGATA, T. Fucoxanthin as the major antioxidant in Hijikia fusiformis, a common edible seaweed. Biosci. Biotechnol. Biochem., v.63, n.3, p.605-607, 1999.

YAN, X.; NAGATA, T.; FAN, X. Antioxidative activities in some common seaweeds. Plant Foods Hum. Nutr., v.52, n.3, p.253-262, 1998 .

YEH, C.T.; YEN, G.C. Induction of hepatic antioxidant enzymes by phenolic acids in rats is accompanied by increased levels of multidrug resistance-associated protein 3 mRNA expression. J. Nutr., v.136, n.1, p.11-15, 2006.

YUAN, Y.V.; WALSH, N.A. Antioxidant and antiproliferative activities of extracts from a variety of edible seaweeds. Food Chem. Toxicol., v. 44, n. 7, p.1144-1150, 2006.

Recebido para publicação em 20 de junho de 2006 Aceito para publicação em 22 de outubro de 2006 